TO APPEAR IN SIAM JOURNAL ON APPLIED DYNAMICAL SYSTEMS (SIADS)

SUBMITTED: 11 DECEMBER 2006; ACCEPTED: 20 JUNE 2007

\title{
CRITICAL POINT ANALYSIS OF TRANSONIC FLOW PROFILES WITH HEAT CONDUCTION
}

\author{
H. DE STERCK*
}

\begin{abstract}
Critical points arising in transonic flow profiles of the steady compressible Euler equations with heat conduction are investigated. Two examples of transonic flow profiles are studied, namely, radial outflow in a gravitational field, and flow in a quasi-one-dimensional nozzle. The stationary flow equations are reformulated in terms of a dynamical system that parametrizes the flow profiles. Adding the heat conduction term introduces a critical point that is of a type different from the well-known sonic critical point that occurs at the transition from subsonic to supersonic flow when there is no heat conduction. This thermal critical point takes over the saddle-point role of the sonic critical point in the flow profile. Both the sonic and the thermal critical points are present in radial outflow profiles, and the type of the sonic critical point is changed from a saddle point to a simple node by the addition of the heat conduction term. In the nozzle case, the sonic point is no longer a critical point of the dynamical system when heat conduction is added. It is illustrated how the results of this analysis can be used for efficient and accurate numerical calculation of transonic flow trajectories and boundary value problems with heat conduction that are of interest in applications like supersonic planetary escape and solar wind models, and in aerospace applications. The analysis also elucidates how many boundary conditions are required for a well-posed transonic boundary value problem with heat conduction, and clarifies the mathematical structure of transonic flow profiles with heat conduction that have been calculated numerically in the literature for various applications.
\end{abstract}

Key words. compressible gas dynamics, transonic flow, heat conduction, critical point

AMS subject classifications. 76H05, 35B38, 65P99

1. Introduction. It is well-known that stationary solutions of the compressible Euler equations of gas dynamics may exhibit critical points where the gas makes a transition from the subsonic to the supersonic flow regime [1,2]. In this paper we investigate the critical points that arise in one-dimensional (1D) transonic flow profiles when heat conduction is added to the Euler equations.

The purpose of this analysis is two-fold.

First, the critical point analysis leads to a better understanding of the mathematical structure of transonic flow profiles with heat conduction that have been calculated numerically in the literature for various applications, for example, supersonic gas escape from planets like Venus, Pluto and the early Earth [3, 4, 5, 6], and extrasolar planets [7]. It also elucidates how many boundary conditions are required for a well-posed transonic boundary value problem with heat conduction.

Second, the results of this critical point analysis can be used for efficient and accurate numerical calculation of transonic flow trajectories and boundary value problems with heat conduction that are of interest in applications like supersonic planetary escape and solar wind models, and in aerospace applications.

In the context of $1 \mathrm{D}$ flow profiles, critical points are points at which the ordinary differential equation (ODE) system that governs the stationary flow is singular, i.e., the derivatives of some of the flow variables cannot be determined from the ODE system at the critical points. The stationary flow equations can be reformulated in terms of a dynamical system that parametrizes the flow profiles. Critical (i.e.,

* Department of Applied Mathematics, University of Waterloo, 200 University Avenue West, Waterloo, Ontario, N2L 3G1, Canada (hdesterck@uwaterloo.ca). 
singular) points of the flow profiles are also critical points of this associated dynamical system in accordance with the standard definition of critical point in a dynamical system, i.e., they are equilibrium solutions of the dynamical system.

In this paper, two examples of $1 \mathrm{D}$ transonic flow profiles are studied, namely, radial outflow in a gravitational field [8], and flow in a quasi-one-dimensional nozzle [9]. The properties of the critical points that arise in these kinds of flows are wellknown for the Euler model without heat conduction. Here, we investigate the critical points that arise when heat conduction is added to the Euler equations.

In the first part of the paper we study such transonic flow solutions for the case of radial spherically symmetric outflow from spherical objects with a gravitational field. The nozzle application is discussed subsequently.

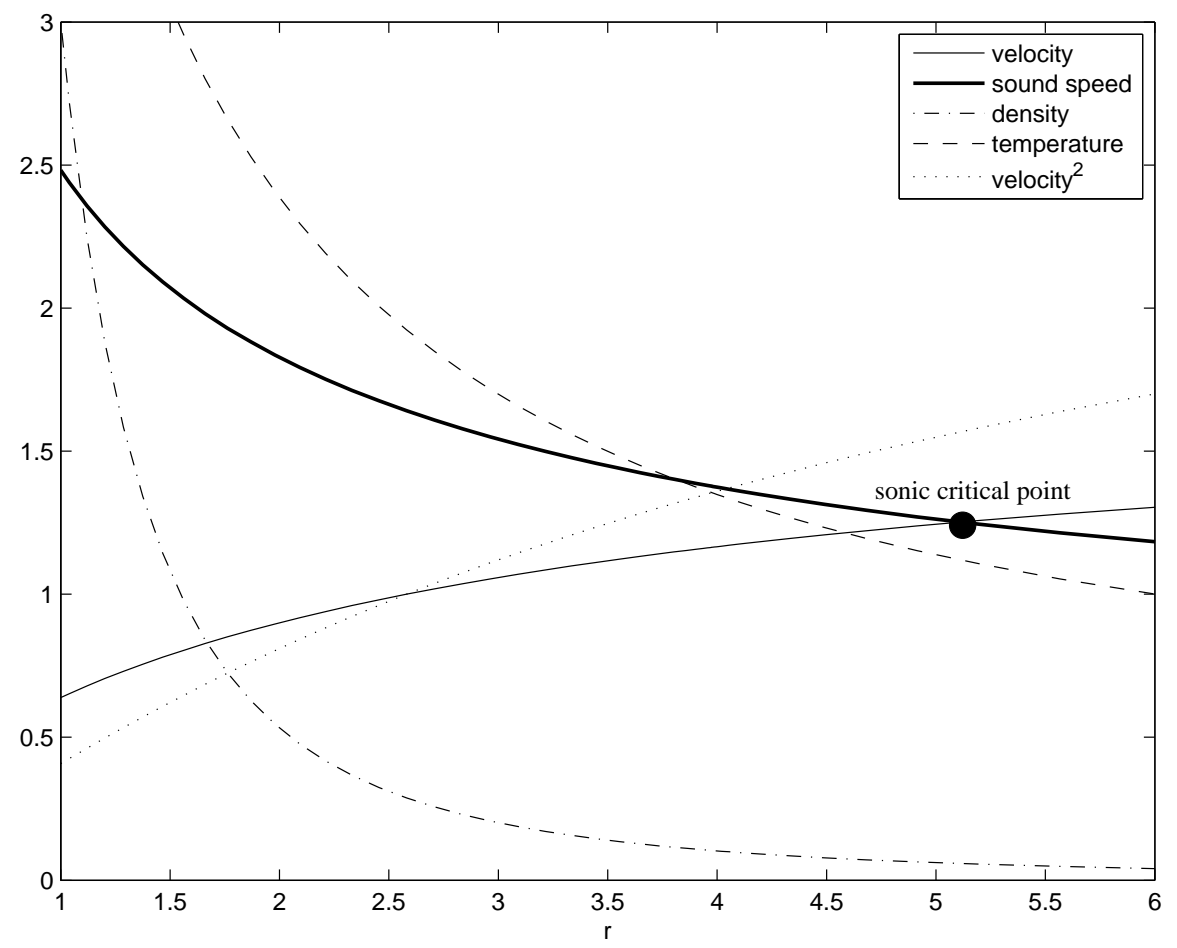

FIG. 1.1. Radial transonic flow solution for $G M=15, q_{\text {heat }}(r) \equiv 0$ and $\kappa(T)=(T / 5)^{0.72}$, and with boundary conditions $\rho=3, T=4.4$ and $d T / d r=-4$ at the inflow boundary $r_{a}=1$. The sonic critical point is indicated.

Fig. 1.1 shows a stationary transonic radial flow solution of the spherically symmetrical Euler equations with radial velocity and heat conduction in a gravitational field, which are given by

$$
\begin{gathered}
\frac{\partial}{\partial t}\left[\begin{array}{c}
\rho r^{2} \\
\rho u r^{2} \\
\left(\frac{p}{\gamma-1}+\frac{\rho u^{2}}{2}\right) r^{2}
\end{array}\right]+\frac{\partial}{\partial r}\left[\begin{array}{c}
\rho u r^{2} \\
\rho u^{2} r^{2}+p r^{2} \\
\left(\frac{\gamma p}{\gamma-1}+\frac{\rho u^{2}}{2}\right) u r^{2}
\end{array}\right]= \\
0 \\
{\left[\begin{array}{c}
0 G M+2 p r \\
-\rho G M u+q_{\text {heat }} r^{2}+\frac{\partial}{\partial r}\left(\kappa r^{2} \frac{\partial T}{\partial r}\right)
\end{array}\right] .}
\end{gathered}
$$


Here, $\rho$ is the fluid mass density, $p$ is the pressure, $u$ is the radial velocity, $r$ is the radial coordinate, $t$ is time, $-\rho G M / r^{2}$ is the gravitational force density, and $\gamma>1$ is the adiabatic constant. With $G$ being the universal gravity constant and $M$ the mass of the outflow object, we take $G M=15$ in normalized units for all the test problems in this paper. The right hand side of the equation contains a heat source term $q_{\text {heat }}$, and a heat conduction term with conductivity $\kappa$. This set of equations is used as a model for supersonic gas escape from Venus, Pluto, the early Earth, and extrasolar planets $[3,4,5,6,7]$, and is related to models for the solar wind $[8,10,11]$.

The temperature $T$, sound speed $c$, entropy $S$ and radial mass flux $F$ are defined by

$$
\begin{aligned}
T & =\frac{p}{\rho}, \\
c^{2} & =\frac{\gamma p}{\rho}=\gamma T, \\
S & =\frac{p}{\rho^{\gamma}}, \\
F & =\rho u r^{2},
\end{aligned}
$$

in our choice of nondimensional units. In general, the heat source $q_{\text {heat }}$ and heat conductivity $\kappa$ can be functions of the radius $r$ and/or the gas state variables $\rho, p$ and $u$. In particular, we consider radial dependence of the heat source $q_{\text {heat }}(r)$ and temperature dependence of the heat conductivity $\kappa(T)$, which are assumptions that are physically relevant for the applications we target $[3,4,5,6,7]$. Fig. 1.1 shows a stationary transonic solution to Eq. (1.2) with the radial outflow velocity from the planet starting out subsonically near the left boundary. The critical point at the transition to supersonic flow is indicated.

Stationary transonic solutions to the Euler equations are notoriously hard to compute numerically. One of the goals of our critical point analysis is to provide results that allow to numerically calculate radial transonic flows with heat conduction efficiently and accurately. Traditional techniques employ time-marching strategies of the time-dependent equations $[12,13]$. However, explicit time-marching approaches tend to converge very slowly, while implicit techniques are expensive per iteration and may suffer from robustness problems. On the other hand, solving the stationary equations directly has proved difficult due to the presence of critical points of saddle point type and the associated difficulties with the changing nature of the solution at the critical points, and with the number of boundary conditions to be imposed. In [14] we have proposed a new numerical algorithm for efficiently and accurately calculating radial transonic flows for the Euler equations, which is based on a dynamical systems approach. The results from the present paper allow to apply these methods to the case where heat conduction is added to the Euler equations.

This paper is structured as follows. In the next section we recall results on critical points for radial Euler flows in a gravitational field without heat conduction, followed by a critical point analysis for the case when heat conduction is added to the Euler model. In Section 3 it is shown how the analysis can be extended to the case of a quasi-1D converging-diverging nozzle. Section 4 illustrates how the results on critical points can be utilized for efficient direct calculation of transonic flow trajectories and boundary value problems for the case of radial outflow in a gravitational field. Conclusions are formulated in Section 5. 
2. Critical point analysis of radial outflow in a gravitational field. The derivations to be presented in this section start out from rewriting the stationary Euler equations in spherical symmetry

$$
\frac{d}{d r}\left[\begin{array}{c}
\rho u r^{2} \\
\rho u^{2} r^{2}+p r^{2} \\
\left(\frac{\gamma p}{\gamma-1}+\frac{\rho u^{2}}{2}\right) u r^{2}
\end{array}\right]=\left[\begin{array}{c}
0 \\
-\rho G M+2 p r \\
-\rho G M u+q_{\text {heat }} r^{2}+\frac{\partial}{\partial r}\left(\kappa r^{2} \frac{\partial T}{\partial r}\right)
\end{array}\right]
$$

as an ODE system with unknowns $F(r), u(r)$ and $T(r)$ and with decoupled first derivatives, as follows:

$$
\begin{aligned}
\frac{d F}{d r}= & 0 \\
\frac{d u}{d r}= & \frac{1}{r^{2}\left(u^{2}-c^{2}\right)}\left(2 u c^{2}\left(r-\frac{G M}{2 c^{2}}\right)-(\gamma-1) \frac{q_{\text {heat }} r^{4} u}{F}\right. \\
& \left.-(\gamma-1) \frac{r^{2} u}{F} \frac{d}{d r}\left(\kappa r^{2} \frac{d T}{d r}\right)\right) \\
\frac{d T}{d r}= & \frac{\gamma-1}{r^{2}\left(u^{2}-c^{2}\right)}\left(T\left(G M-2 u r^{2}\right)-\frac{q_{h e a t} r^{4}\left(T-u^{2}\right)}{F}\right. \\
& \left.-\frac{r^{2}\left(T-u^{2}\right)}{F} \frac{d}{d r}\left(\kappa r^{2} \frac{d T}{d r}\right)\right) .
\end{aligned}
$$

It should be noted that the derivations to be presented can also be performed using other state variables, for instance the density $\rho$ or the entropy $S$. The choice of variables somewhat influences the effort required to obtain the results and the simplicity of some of the resulting expressions, but in the end the results and conclusions are the same regardless of the choice of variables. The variables in system (2.2) were chosen because they are of direct interest in the applications targeted.

2.1. Euler equations without heat conduction. We first recall results on critical points and their types for the case of vanishing heat conduction $\kappa(T) \equiv 0$. In this case, system (2.2) reduces to a decoupled first-order ODE system, which can be rewritten as an autonomous system by introducing the independent variable $s$ that parametrizes the solution trajectories. This leads to the dynamical system

$$
\begin{aligned}
& \frac{d r}{d s}=r^{2}\left(u^{2}-c^{2}\right), \\
& \frac{d F}{d s}=0, \\
& \frac{d u}{d s}=2 u c^{2}\left(r-\frac{G M}{2 c^{2}}\right)-(\gamma-1) q_{\text {heat }} \frac{r^{4} u}{F}, \\
& \frac{d T}{d s}=(\gamma-1) T\left(G M-2 u^{2} r\right)-(\gamma-1) q_{\text {heat }} \frac{r^{4}}{F}\left(T-u^{2}\right) .
\end{aligned}
$$

In general terms, the autonomous system is denoted by

$$
\frac{d \mathbf{V}}{d s}=\mathbf{G}(\mathbf{V})
$$


with state vector $\mathbf{V}=[F(s) u(s) r(s) T(s)]^{T}$. The critical points (or equilibrium points) of dynamical system (2.3) satisfy the two conditions

$$
\begin{aligned}
& u_{\text {crit }}=\sqrt{\gamma T_{\text {crit }}}=c_{\text {crit }}, \\
& T_{\text {crit }}=\frac{G M}{2 \gamma r_{\text {crit }}}+(\gamma-1) \frac{q_{\text {heat }} r_{\text {crit }}^{3}}{2 \gamma F_{\text {crit }}} .
\end{aligned}
$$

Condition (2.5) identifies the critical point as the well-known sonic critical point. Note that the dynamical system as formulated here is degenerate, in that the fourdimensional (4D) system allows for a two-dimensional (2D) manifold of critical points, as there are only two conditions to be fulfilled in stead of four.

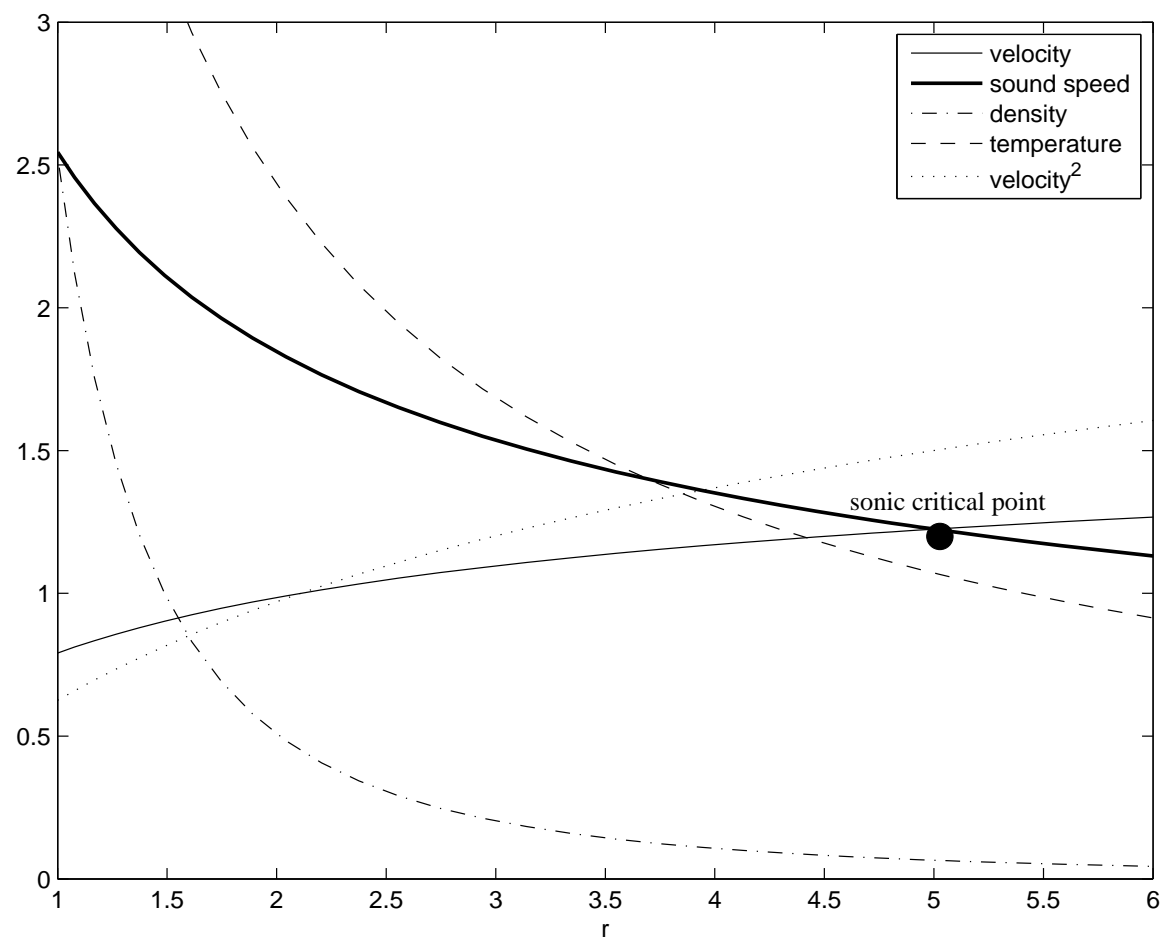

FIG. 2.1. Radial transonic flow solution for $q_{\text {heat }}(r) \equiv 0$ and $\kappa(T) \equiv 0$. The critical radius $r_{\text {crit }}=5$, and the flux $F=2$. The sonic critical point is indicated.

The type of the critical point can be derived by investigating the eigenvalues of the Jacobian matrix

$$
\left.\frac{\partial \mathbf{G}}{\partial \mathbf{V}}\right|_{\mathbf{V}_{\text {crit }}}
$$

For the case of vanishing heating source term $q_{\text {heat }}(r) \equiv 0$, we obtain

$$
\begin{aligned}
& \lambda_{1}=0, \\
& \lambda_{2}=0, \\
& \lambda_{3}=G M \sqrt{(-3 \gamma+5) / 2}, \\
& \lambda_{4}=-G M \sqrt{(-3 \gamma+5) / 2} .
\end{aligned}
$$


When $\gamma<5 / 3$, this leads to a (degenerate) saddle point. For example, for the outflow problem of hydrogen gas from planetary atmosphere that we target $[3,4,5,6,7]$, the value $\gamma=7 / 5$ for di-atomic gases applies. All the example flow solutions presented in this paper use the value $\gamma=7 / 5$. For general heat source functions $q_{\text {heat }}(r)$, the expressions for Jacobian (2.6) and its eigenvalues are complicated and the detailed expressions are lengthy and uninsightful. However, using symbolical manipulation software, it can easily be verified that the eigenvalues satisfy

$$
\begin{aligned}
& \lambda_{1}=0, \\
& \lambda_{2}=0, \\
& \lambda_{3}=a+\sqrt{b}, \\
& \lambda_{4}=a-\sqrt{b} .
\end{aligned}
$$

Here, $a$ and $b$ are generic placeholders for nonlinear functions that involve the state variables, parameters like $\gamma$ and $G M$, and the heat source function $q_{\text {heat }}(r)$ and its radial derivative $d q_{\text {heat }}(r) / d r$. Depending on the values of these, the type of the critical point changes. Again, the general conditions that determine the type of the critical point are lengthy and do not provide specific insight. We suffice by saying that in many parameter regimes of interest for the applications we target, the critical point turns out to be of (degenerate) saddle point type.

Fig. 2.1 shows an example of a stationary transonic solution to Eq. (1.2) with $\kappa(T) \equiv 0$ and $q_{\text {heat }} \equiv 0$. The critical point at the transition to supersonic flow is indicated. In accordance with the discussion above, the critical point turns out to be of saddle point type.

2.2. Euler equations with heat conduction. We now proceed with the main topic of this paper, namely the critical point analysis of radial transonic Euler flow with heat conduction, i.e., the case where $\kappa(T) \neq 0$. We start from the ODE system (2.2), and define the additional variable

$$
\phi=\kappa r^{2} \frac{d T}{d r}
$$

which is related to the temperature gradient. With the use of this gradient variable $\phi$ we can rewrite ODE system (2.2) as the fully decoupled first-order system

$$
\begin{aligned}
\frac{d F}{d r}= & 0, \\
\frac{d u}{d r}= & \frac{1}{r^{2}\left(u^{2}-c^{2}\right)}\left(2 u c^{2}\left(r-\frac{G M}{2 c^{2}}\right)+\frac{u \phi\left(u^{2}-c^{2}\right)}{\kappa\left(T-u^{2}\right)}\right. \\
& \left.-\frac{(\gamma-1) u T\left(G M-2 u^{2} r\right)}{T-u^{2}}\right), \\
\frac{d T}{d r}= & \frac{\phi}{\kappa r^{2}}, \quad \frac{-\phi F\left(u^{2}-c^{2}\right)}{(\gamma-1) \kappa r^{2}\left(T-u^{2}\right)}+\frac{F T\left(G M-2 u^{2} r\right)}{r^{2}\left(T-u^{2}\right)}-q_{h e a t} r^{2} .
\end{aligned}
$$

This system can be written as an autonomous system by introducing the independent variable $s$ that parametrizes the solution trajectories, resulting in the dynamical 
system

$$
\begin{aligned}
\frac{d r}{d s}= & -r^{2}\left(u^{2}-c^{2}\right)\left(u^{2}-T\right), \\
\frac{d F}{d s}= & 0, \\
\frac{d u}{d s}= & -2 u c^{2}\left(r-\frac{G M}{2 c^{2}}\right)\left(u^{2}-T\right)+\frac{\phi u\left(u^{2}-c^{2}\right)}{\kappa} \\
& -(\gamma-1) u T\left(G M-2 u^{2} r\right), \\
\frac{d T}{d s}= & \frac{-\phi\left(u^{2}-c^{2}\right)\left(u^{2}-T\right)}{\kappa}, \\
\frac{d \phi}{d s}= & \frac{-\phi F\left(u^{2}-c^{2}\right)^{2}}{(\gamma-1) \kappa}+F T\left(G M-2 u^{2} r\right)\left(u^{2}-c^{2}\right) \\
& +q_{\text {heat }} r^{4}\left(u^{2}-c^{2}\right)\left(u^{2}-T\right) .
\end{aligned}
$$

Interestingly, the dynamical system now turns out to have two types of critical points.

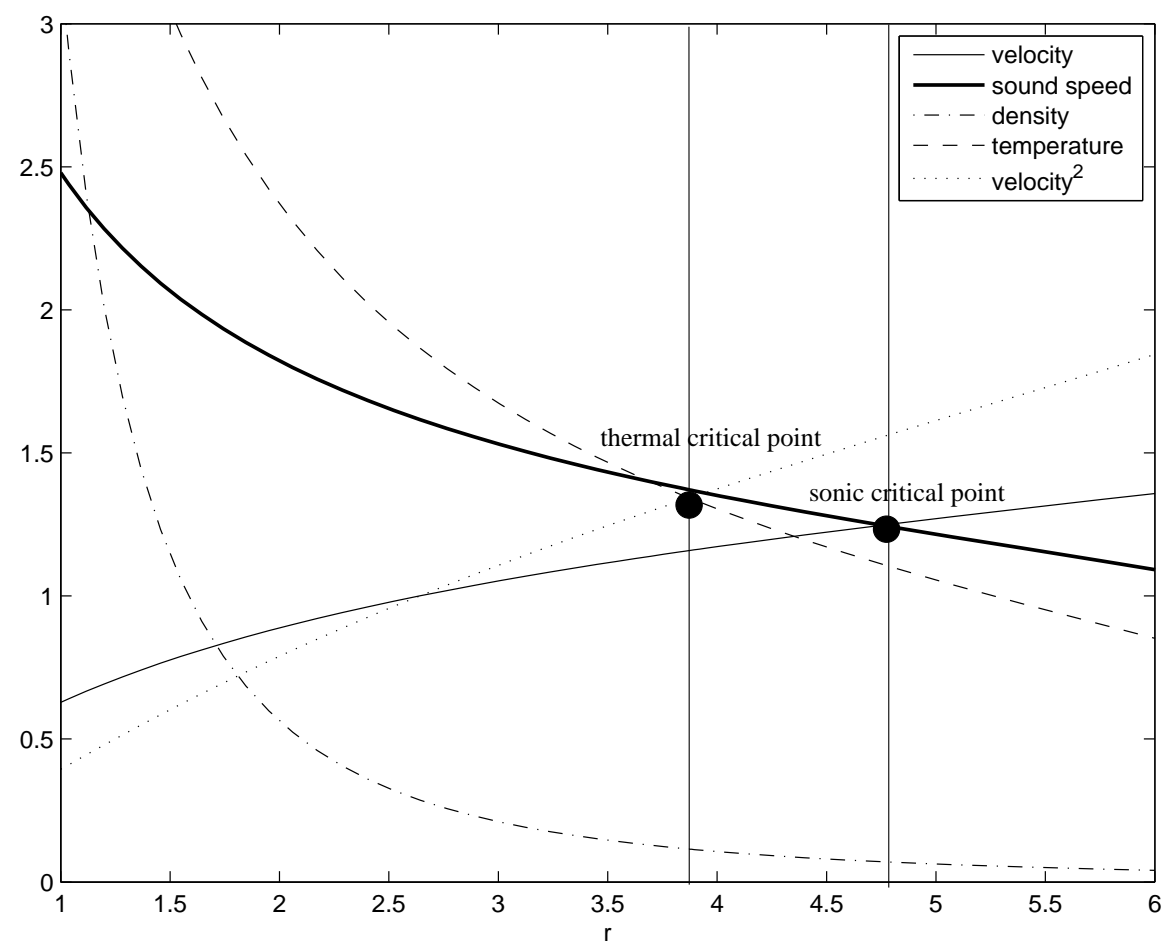

FIG. 2.2. Radial transonic flow solution for $q_{\text {heat }}(r) \equiv 0$ and $\kappa(T) \equiv(T / 5)^{0.72}$. The two critical points are indicated. The rightmost critical point is the well-known sonic critical point, while the leftmost critical point is the thermal critical point that is introduced by including the heat conduction term. The location of the thermal critical point $r_{\text {crit }} \approx 3.87$, its temperature $T \approx 1.34$, and its velocity $u \approx 1.16$.

The first type of critical point is the well-known sonic critical point. This critical 
point is four-fold degenerate, as there is now only one condition at this critical point:

$$
u_{\text {crit }}=\sqrt{\gamma T_{\text {crit }}}=c_{\text {crit }} .
$$

The eigenvalues of the Jacobian are given by

$$
\begin{aligned}
& \lambda_{1}=0 \\
& \lambda_{2}=0 \\
& \lambda_{3}=0 \\
& \lambda_{4}=0 \\
& \lambda_{5}=u_{\text {crit }}^{2}\left(\frac{(\gamma+1) \phi_{\text {crit }}}{\kappa\left(T_{\text {crit }}\right)}+2 G M-\frac{4 u_{c r i t}^{2} r_{\text {crit }}}{\gamma}\right),
\end{aligned}
$$

indicating a critical point of (degenerate) simple node type.

The second type of critical point is characterized by the conditions

$$
\begin{aligned}
u_{c r i t} & =\sqrt{T_{c r i t}}=c_{c r i t} / \sqrt{\gamma}, \\
\frac{\phi_{c r i t}}{\kappa}+G M-2 u_{c r i t}^{2} r_{c r i t} & =0 .
\end{aligned}
$$

We call this type of critical point a thermal critical point $\left(u_{c r i t}^{2}=T_{\text {crit }}\right)$, in order to distinguish it from the sonic critical point type, where $u_{c r i t}^{2}=\gamma T_{\text {crit }}$. Note that the dynamical system is again degenerate, in that the five-dimensional (5D) system allows for a three-dimensional (3D) manifold of critical points, as there are only two conditions to be fulfilled in stead of five.

For this critical point, the eigenvalues of the Jacobian are given by

$$
\begin{aligned}
& \lambda_{1}=0, \\
& \lambda_{2}=0, \\
& \lambda_{3}=0, \\
& \lambda_{4}=a+\sqrt{b}, \\
& \lambda_{5}=a-\sqrt{b},
\end{aligned}
$$

where $a$ and $b$ are generic placeholders for nonlinear functions that involve the state variables, parameters like $\gamma$ and $G M$, the heat source function $q_{\text {heat }}(r)$ and its radial derivative $d q_{\text {heat }}(r) / d r$, and the heat conduction function $\kappa(T)$ and its temperature derivative $d \kappa(T) / d T$. Full expressions for $a$ and $b$ can be derived using symbolical manipulation software, but they are cumbersome and do not lead to further insight. Again, depending on the values of the parameters, the type of the critical point changes. As before, the general conditions that determine the type of the critical point are lengthy and do not provide specific insight, and we suffice by saying that in many parameter regimes of interest for the applications we target, the critical point turns out to be of (degenerate) saddle point type.

We can thus conclude that, interestingly, adding the heat conduction term to the Euler model changes the type of the sonic critical point from a saddle point to a simple node, and introduces an additional, thermal, critical point into the system, which is of saddle point type. It turns out that transonic solutions pass through the two types of critical points, as illustrated by the following example flow solution.

Fig. 2.2 shows an example of a stationary transonic solution to Eq. (1.2) with $\kappa(T)=(T / 5)^{0.72}$, and $q_{\text {heat }}=0$. The exponent 0.72 for the heat conduction function 
is chosen for this example flow solution due to its relevance for planetary atmosphere calculations $[3,4,5,6,7]$. The two critical points are indicated on the figure. The leftmost, thermal, critical point occurs where $u=\sqrt{T}$, and is of saddle point type. The rightmost critical point is the sonic critical point, where $u=\sqrt{\gamma T}$.

The flow profile of Fig. 2.2 thus has two critical points, but they are of different type (one is thermal, and the other is sonic). Note that this situation is different from the multitransonic accretion profiles studied in [15], in which multiple critical points occur that are all of sonic type, for flow equations without heat conduction.

3. Critical point analysis of quasi-1D nozzle flow. In this Section we repeat the analysis presented above for the case of a quasi-1D converging-diverging nozzle [9], leading to similar results. Fig. 3.1 shows a de Laval nozzle, in which subsonic flow can be accelerated to supersonic flow in a continuous fashion. If the cross-sectional flow area $A(x)$ varies slowly, the nozzle is long and slender, and cross-flow velocities are small, the flow can be modeled as quasi-1D (see, e.g., [9]), leading to the following equation system:

$$
\begin{array}{r}
\frac{\partial}{\partial t}\left[\begin{array}{c}
\rho A \\
\rho u A \\
\left(\frac{p}{\gamma-1}+\frac{\rho u^{2}}{2}\right) A
\end{array}\right]+\frac{\partial}{\partial x}\left[\begin{array}{c}
\rho u A \\
\rho u^{2} A+p A \\
\left(\frac{\gamma p}{\gamma-1}+\frac{\rho u^{2}}{2}\right) u A
\end{array}\right]= \\
{\left[\begin{array}{c}
0 \\
p \frac{d A}{d x} \\
\frac{\partial}{\partial x}\left(\kappa A \frac{\partial T}{\partial x}\right)
\end{array}\right] .}
\end{array}
$$

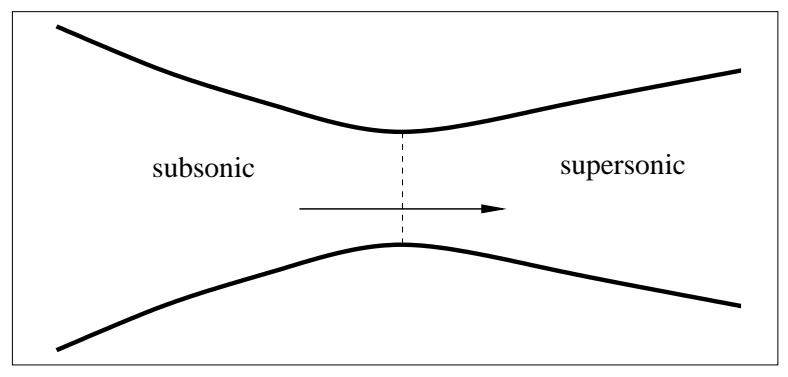

FIG. 3.1. Transonic flow in a converging-diverging nozzle.

3.1. Nozzle flow without heat conduction. First, consider the case that the heat conduction coefficient, $\kappa(T)$, vanishes. Then the dynamical system associated with the stationary part of Eq. (3.2) can be derived as above, leading to

$$
\begin{aligned}
& \frac{d x}{d s}=u^{2}-c^{2}, \\
& \frac{d F}{d s}=0, \\
& \frac{d u}{d s}=\frac{\gamma u T}{A} \frac{d A}{d x}, \\
& \frac{d T}{d s}=-\frac{(\gamma-1) u^{2} T}{A} \frac{d A}{d x} .
\end{aligned}
$$


The critical points of dynamical system (3.2) satisfy the two conditions

$$
\begin{aligned}
u_{\text {crit }} & =\sqrt{\gamma T_{\text {crit }}}=c_{\text {crit }}, \\
\frac{d A}{d x}\left(x_{\text {crit }}\right) & =0 .
\end{aligned}
$$

This dynamical system has, thus, a sonic critical point, which can only occur at the throat of the nozzle, where $d A(x) / d x=0$. The type of the critical point can be determined by investigating the eigenvalues of the Jacobian matrix, which are given by

$$
\begin{aligned}
& \lambda_{1}=0, \\
& \lambda_{2}=0, \\
& \lambda_{3}=\sqrt{\frac{\gamma+1}{A} \frac{d^{2} A}{d x^{2}}} u^{2}, \\
& \lambda_{4}=-\sqrt{\frac{\gamma+1}{A} \frac{d^{2} A}{d x^{2}}} u^{2} .
\end{aligned}
$$

This shows that the sonic critical point is a saddle point at the throat of the nozzle (where $d^{2} A(x) / d x^{2} \geq 0$ ).

3.2. Nozzle flow with heat conduction. When heat conduction is added to the Euler model $(\kappa(T) \neq 0)$, we can define the additional variable

$$
\phi=\kappa \frac{d T}{d x} .
$$

With the use of this gradient variable $\phi$, we can, as before, derive the dynamical system

$$
\begin{aligned}
& \frac{d x}{d s}=u^{2}-T, \\
& \frac{d F}{d s}=0, \\
& \frac{d u}{d s}=\frac{u T}{A} \frac{d A}{d x}-\frac{u \phi}{\kappa}, \\
& \frac{d T}{d s}=\frac{\phi}{\kappa}\left(u^{2}-T\right), \\
& \frac{d \phi}{d s}=\left(\frac{F u^{2} T}{A^{2}}-\frac{\phi}{A}\left(u^{2}-T\right)\right) \frac{d A}{d x}+\frac{F \phi}{A \kappa} \frac{u^{2}-\gamma T}{\gamma-1} .
\end{aligned}
$$

Contrary to the radial outflow case with heat conduction, this dynamical system has only one type of critical point. The critical point is characterized by the conditions

$$
\begin{aligned}
u_{\text {crit }} & =\sqrt{T_{\text {crit }}}, \\
\frac{1}{A\left(x_{\text {crit }}\right)} \frac{d A}{d x}\left(x_{\text {crit }}\right) & =\frac{\phi_{\text {crit }}}{\kappa T_{\text {crit }}}=\frac{1}{T_{\text {crit }}} \frac{d T}{d x}\left(x_{\text {crit }}\right) .
\end{aligned}
$$


This critical point is a thermal critical point $\left(u_{c r i t}^{2}=T_{\text {crit }}\right)$. It is interesting to note that the thermal critical point does not occur at the throat of the nozzle. For this critical point, the eigenvalues of the Jacobian are given by

$$
\begin{aligned}
\lambda_{1} & =0, \\
\lambda_{2} & =0, \\
\lambda_{3} & =0, \\
\lambda_{4} & =a+\sqrt{b}, \\
\lambda_{5} & =a-\sqrt{b},
\end{aligned}
$$

where $a$ and $b$ are generic placeholders for nonlinear functions that involve the state variables, the parameter $\gamma$, the area function $A(x)$ and its first and second spatial derivatives, and the heat conduction function $\kappa(T)$ and its temperature derivative $d \kappa(T) / d T$. Again, full expressions for $a$ and $b$ can be derived using symbolical manipulation software, but they are cumbersome and do not lead to further insight. Also, depending on the values of the parameters, the type of the critical point changes. As before, the general conditions that determine the type of the critical point are lengthy and do not provide specific insight, and we suffice again by saying that in many parameter regimes of interest, the critical point turns out to be of (degenerate) saddle point type.

We can, thus, conclude that adding the heat conduction term to the Euler model here removes the sonic critical point as a critical point altogether, and introduces a new thermal critical point of saddle point type.

The result is, thus, similar to the radial outflow case: adding heat conduction to the Euler equations results in the appearance of a thermal critical point. The thermal critical point takes over the role of the sonic critical point as a saddle point in the flow profile.

4. Numerically calculated example flow solutions for radial outflow in a gravitational field. In this section, we illustrate how the critical point properties derived above can be used for efficiently calculating stationary transonic Euler flows numerically, for the case of radial outflow in a gravitational field. Note that example flow solutions for the quasi-1D nozzle application can be calculated in a similar manner.

We consider two typical situations. In the first, most simple, case, the state variables at the critical point are known, and a numerical approximation to the critical trajectory through the critical point is sought. In the second case, some state variables are known at the left inflow boundary, and a numerical approximation to the transonic solution that matches the boundary conditions is sought. Such a critical boundary value problem (BVP) is significantly more complicated to solve, but here too the knowledge about the critical points that was developed aids in efficiently obtaining a numerical approximation. This section also serves to illustrate how the addition of heat conduction influences transonic Euler solutions.

4.1. Example trajectories. We first recall why trajectories that pass through critical points of saddle point type are difficult to approximate numerically. This can be explained most easily for the simplified case of isothermal Euler flow $[8,11,14]$. In this case the sound speed $c$ is a constant, and a decoupled ODE for $u(r)$ can be 


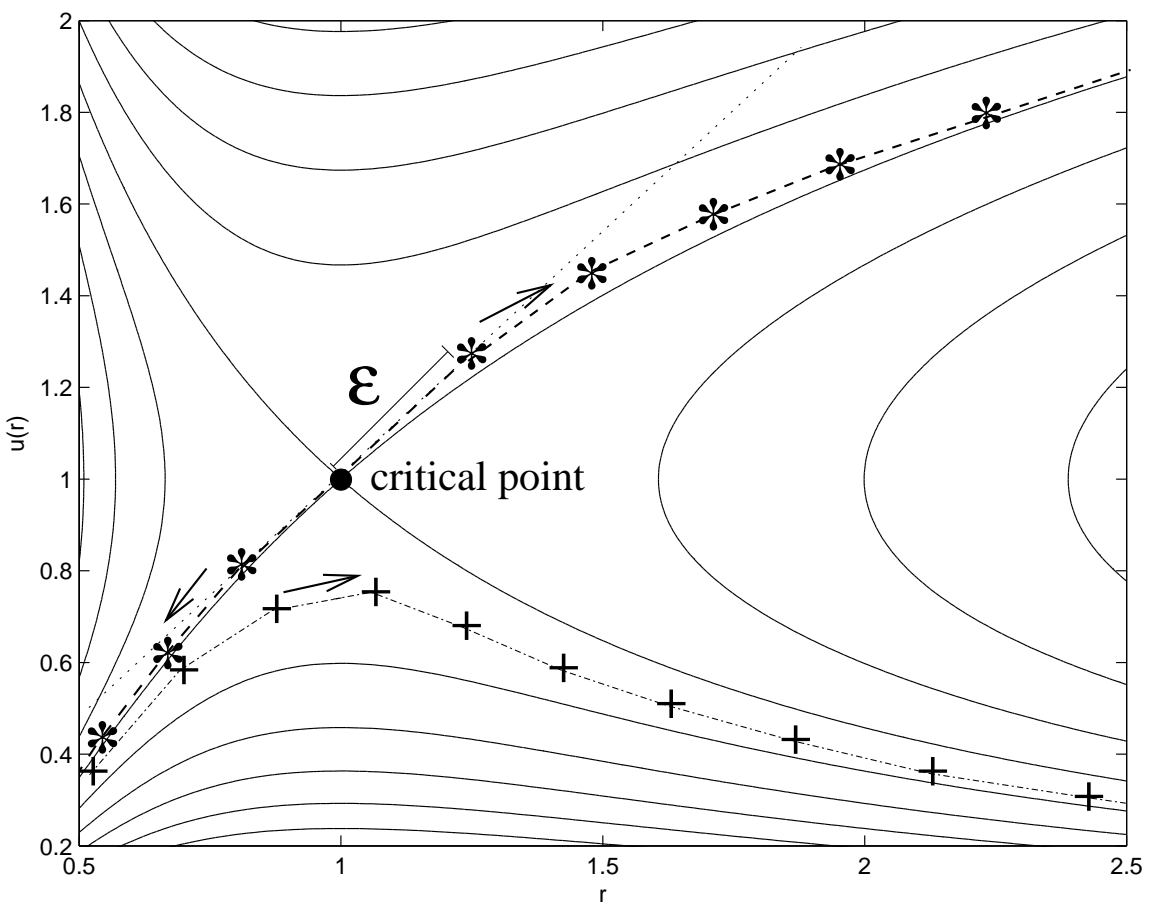

FIG. 4.1. Solution curves $u(r)$ for the spherically symmetric isothermal Euler eqations with $G M=2$ and $c=1$. Numerical integration from the left boundary (dash-dotted curve) does not allow to approximate the transonic solution, but integration in two directions outward from the critical point allows numerical approximation of the transonic solution. The arrows indicate the direction of numerical integration.

derived

$$
\frac{d u}{d r}=\frac{\left.2 u c^{2}\left(r-\frac{G M}{2 c^{2}}\right)\right)}{r^{2}\left(u^{2}-c^{2}\right)},
$$

which leads to the simplified dynamical system

$$
\frac{d u(s)}{d s}=-2 u c^{2}\left(r-\frac{G M}{2 c^{2}}\right),
$$

$$
\frac{d r(s)}{d s}=-r^{2}\left(u^{2}-c^{2}\right) .
$$

This system features a critical point of saddle point type that is specified by the conditions

$$
\begin{aligned}
& r_{\text {crit }}=\frac{G M}{2 c^{2}}, \\
& u_{\text {crit }}=c,
\end{aligned}
$$

or the point $(1,1)$ in the $(r, u)$ phase plane for the choice of parameters $G M=2$ and $c=1$. Fig. 4.1 shows solution trajectories in the $(r, u)$ phase plane. 

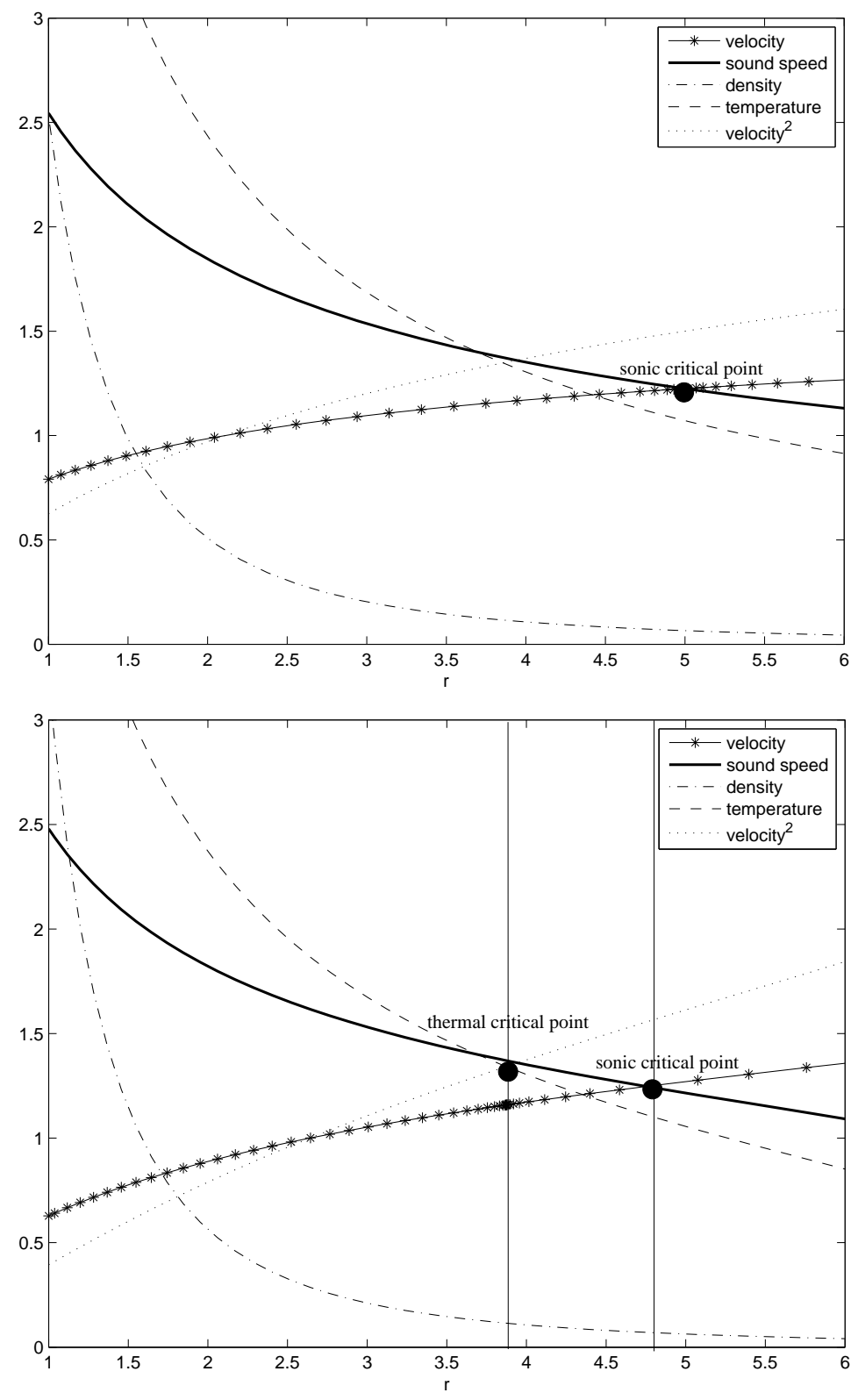

FIG. 4.2. Radial outflow trajectories without and with heat conduction. Top: Radial transonic flow solution for $q_{\text {heat }}(r) \equiv 0$ and $\kappa(T) \equiv 0$. The sonic critical radius $r_{\text {crit }}=5$, and the flux $F=2$. Bottom: Radial transonic flow solution for $q_{\text {heat }}(r) \equiv 0$ and $\kappa(T) \equiv(T / 5)^{0.72}$. The thermal critical point has critical radius $r_{\text {crit }} \approx 3.87$, temperature $T \approx 1.34$, and velocity $u \approx 1.16$.

Assume that one wants to find a numerical approximation for the transonic solution that passes through the critical point. It is easy to see that numerical integration from the left does not lead to a desirable result, as numerical errors cause the approximation to deviate from the transonic curve. However, integration in two directions outward from the critical point (dashed curves) leads to an accurate numerical ap- 
proximation of the transonic curve. The initial points for the outward integrations should be taken in the direction of the tangent to the transonic solution, which is an eigenvector of the Jacobian matrix $\partial G(V) / \partial V$ at the critical point. This approach for calculating the stable or unstable manifold of a saddle point is a standard dynamical systems technique [16], and can be applied to transonic Euler flow calculation once the critical point location and state are known.

Fig. 4.2 illustrates how this approach can be used for numerical approximation of transonic Euler flow with and without heat conduction. The top panel shows Euler flow without heat conduction, with critical radius $r_{\text {crit }}=5$ and flux $F=2$. This is the same solution as shown in Fig. 2.1, but now we have added the numerical integration points that were used by our ODE integrator. Numerical integration is outward from the sonic critical point, which is of saddle point type. We have used an adaptive fourth-fifth order accurate Runge-Kutta ODE integrator for the result shown, namely the RK45 Fehlberg method as described, for example, in [17]. This solution has $u^{2}=T \approx 1.34$ at $r \approx 3.87$.

The top flow profile has to be compared with the solution presented in the bottom panel, which features heat conduction, and has the same values for temperature and velocity as the top solution at $r \approx 3.87$. This is the same solution as shown in Fig. 2.2 , but is repeated here for the purpose of comparison with the top panel. Numerical integration is now outward from the thermal critical point, and it can be seen that the numerical integration does not experience any problem at the sonic critical point, which is now a simple node due to the presence of heat conduction. The top and bottom solutions both feature $u^{2}=T \approx 1.34$ at $r \approx 3.87$, but differ otherwise due to the presence of heat conduction in the bottom solution.

4.2. Example Boundary Value Problems. In Fig. 4.3 we compare two BVPs without and with heat conduction. The top solution has $q_{\text {heat }}(r) \equiv 0$ and $\kappa(T) \equiv 0$, while the bottom solution has $q_{\text {heat }}(r) \equiv 0$ and $\kappa(T) \equiv(T / 5)^{0.72}$. Both flow solutions have $\rho=3$ and $T=4.4$ at the inflow boundary. In addition, the bottom solution, with heat conduction, requires one more boundary condition at the inflow boundary in order for the BVP to be well-posed; $d T / d r=-4$ is imposed additionally at the lower boundary. Note the difference in scale of the two plots: the heat conduction brings the location of the sonic critical point much closer to the inflow boundary. The values of the resulting flux variable $F$, which is the main quantity of interest for this kind of BVP solutions in the case of planetary outflow calculations $[3,4,5,6,7]$, is also influenced significantly by the heat conduction: $F \approx 0.78$ for the BVP without heat conduction, but $F \approx 1.92$ with heat conduction.

Determining the number of boundary conditions necessary for transonic BVPs is not a trivial matter, and numerical calculation of transonic BVPs is difficult due to the a priori unknown location of the critical points. Recently we have proposed a new algorithm for numerical approximation of transonic BVP solutions to the Euler equations. We suffice here with a brief explanation of how the BVP solutions of Fig. 4.3 were obtained, and full details about the algorithmic approach can be found in [14].

Regarding the issue of the number of boundary conditions, the isothermal Euler case is again illustrative. The solution trajectories of Eqs. (4.1) and (4.2) shown in Fig. 4.1 are uniquely identified by one boundary condition at the left inflow boundary, except for the transonic curve, whose specification does not require any numeric boundary condition value for the velocity $u$ at the left boundary, in the following sense: just the requirement that the solution is transonic, i.e., that the flow is subsonic at 

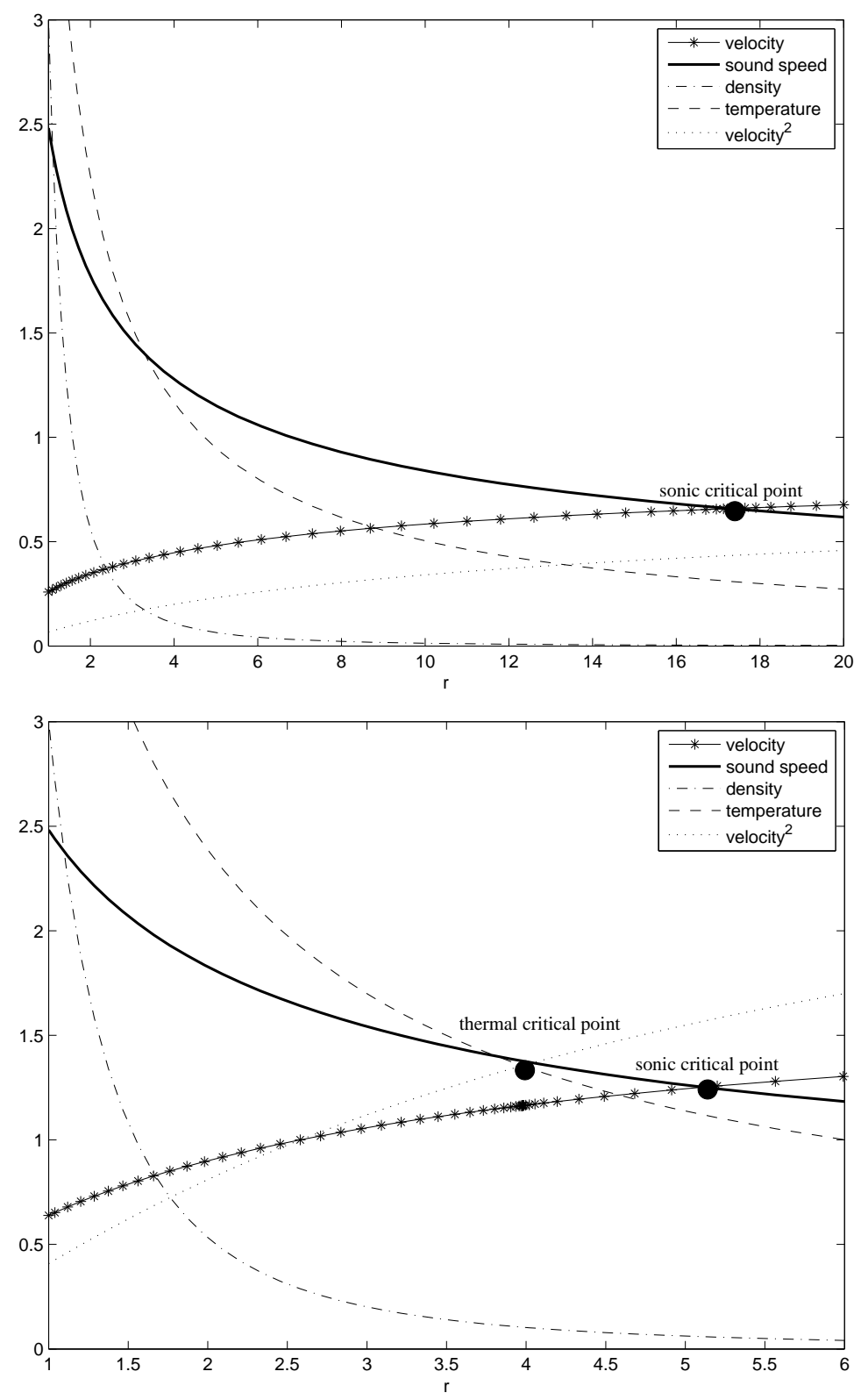

FIG. 4.3. BVP flow trajectories without (top) and with (bottom) heat conduction. Both flow solutions have $\rho=3$ and $T=4.4$ at the inflow boundary. In addition, $d T / d r=-4$ is imposed at the inflow boundary for the bottom solution. The NCP method from [14] was used to solve these BVPs numerically.

the left boundary and supersonic at the right boundary, uniquely determines the solution, and the value of the veolcity $u$ at the inflow boundary follows from the transonic flow requirement. In the same way, transonic Euler solutions without heat conduction require only two boundary conditions for three-dimensional system (2.2) (with $\kappa(T) \equiv 0)$, in addition to the requirement that the flow solution is transonic, and, 
similarly, transonic Euler solutions with heat conduction require only three boundary conditions for four-dimensional system (2.10), again in addition to the requirement that the flow solution is transonic.

The BVP solution can then be approximated numerically in an iterative procedure that uses the numerical integration method outward from the critical points that was described above, combined with a Newton method that allows the critical point to vary within the manifold of possible critical points, thus driving the iterative approximation toward the particular transonic solution and critical point that match the inflow boundary conditions. See [14] for details about this Newton Critical Point algorithm. Indeed, in the case of the Euler equations without heat conduction, the two-fold degeneracy of the sonic critical point of saddle-point type (2.5) conveniently matches the number of inflow boundary conditions for the BVP. In a similar way, the results of this paper show that, in the case of the Euler equations with heat conduction, the three-fold degeneracy of the non-sonic critical point of saddle-point type (2.14) matches the number of inflow boundary conditions that specify the transonic BVP solution uniquely.

5. Conclusions. In this paper, we have presented a critical point analysis for transonic flow solutions of the steady compressible Euler equations with heat conduction. By means of reformulating the stationary ODE system as a first-order system that can be rewritten as a dynamical system, it was shown that the addition of the heat conduction term introduces a new critical point that is different from the wellknown sonic critical point. This so-called thermal critical point is of saddle point type in parameter regimes of interest. For radial outflows, the sonic point remains a critical point, and the flow profile, thus, has two critical points of different type, but the sonic critical point is changed from a saddle point to a simple node by the addition of the heat conduction term. In the quasi-1D nozzle case, the sonic point is not a critical point of the dynamical system anymore when heat conduction is added. Heat conduction adds a term with a second spatial derivative of the temperature, and it can be concluded that adding this heat conduction term changes the critical point properties of the ODE system and the transonic flow solutions significantly.

It was illustrated how the results of this analysis can be used for efficient and accurate numerical calculation of transonic radial outflow trajectories and boundary value problems using the Newton Critical Point method that was introduced in [14]. The analysis also elucidates how many boundary conditions are required for a wellposed transonic boundary value problem with heat conduction. The results presented in this paper, thus, reveal the mathematical structure of stationary transonic solutions of the compressible Euler equations with heat conduction, which are of interest in applications like supersonic gas escape from Venus, Pluto and the early Earth [3, 4, $5,6]$.

In future work, we will study how the phenomena described in this paper play a role in $2 \mathrm{D}$ and $3 \mathrm{D}$ flows with heat conduction, where symmetry is relaxed. We expect that the analysis given here can be extended to these higher-dimensional cases, and we speculate that multiple types of critical points may arise there as well. We also intend to investigate whether numerical methods for stationary transonic flow simulation that are based on dynamical systems analysis may be developed for flow problems in multiple spatial dimensions. This will be addressed in future research. 


\section{REFERENCES}

[1] R. Courant and K. O. Friedrichs, 'Supersonic flow and shock waves', Interscience Publishers, New York, 1948.

[2] R. Courant and D. Hilbert, 'Methods of mathematical physics', Interscience Publishers, New York, Volume 2, 1962.

[3] A. J Watson, T. M. Donahue, and J. C. G. Walker, 'The dynamics of a rapidly escaping atmosphere - Applications to the evolution of earth and Venus', Icarus 48, 150-166, 1981.

[4] J. F. Kasting and J. B. Pollack, 'Loss of water from Venus. I - Hydrodynamic escape of hydrogen', Icarus 53, 479-508,1983.

[5] Feng Tian and Owen B. Toon, 'Hydrodynamic escape of nitrogen from Pluto', Geophys. Res. Lett. 32, 18201-18204, 2005.

[6] Feng Tian, Owen B. Toon, Alexander A. Pavlov, and H. De Sterck, 'A Hydrogen-Rich Early Earth Atmosphere', Science 308, 1014-1017, 2005.

[7] Feng Tian, Owen B. Toon, Alexander A. Pavlov, and H. De Sterck, 'Transonic Hydrodynamic Escape of Hydrogen from Extrasolar Planetary Atmospheres', Astrophysical Journal 621, 1049-1060, 2005.

[8] M. Goossens, 'An Introduction to Plasma Astrophysics and Magnetohydrodynamics', Kluwer, Dordrecht, 2003.

[9] G. I. Benison and E. L. Rubin, 'A time-dependent analysis for quasi-one-dimensional, viscous, heat conducting, compressible Laval nozzle flows', Journal of Engineering Mathematics 5, 39-49, 1970.

[10] R. Keppens and J. P. Goedbloed, 'Numerical simulations of stellar winds: polytropic models', Astron. Astrophys. 343, 251-260, 1998.

[11] T. Sakurai, 'Magnetohydrodynamic solar/stellar wind models', Computer Physics Reports 12: 247-273, 1990.

[12] R.J. LeVeque, 'Finite volume methods for hyperbolic problems', Cambridge University Press, Cambridge, 2002.

[13] B. van Leer, W.T. Lee and P.L. Roe, 'Characteristic time-stepping or local preconditioning of the Euler equations', AIAA Paper 91-1552,1991.

[14] H. De Sterck and F. Tian, 'A Fast and Accurate Algorithm for Computing Radial Transonic Flows', submitted, 2007.

[15] S. Chaudhury, A. K. Ray, and T. K. Das, 'Critical properties and stability of stationary solutions in multitransonic pseudo-Schwarzschild accretion', MNRAS 373, 146-156, 2006.

[16] Bernd Krauskopf, Hinke M. Osinga, Eusebius J. Doedel, Michael E. Henderson, John M. Guckenheimer, Alexander Vladimirsky, Michael Dellnitz and Oliver Junge, 'A survey of methods for computing (un)stable manifolds of vector fields', Int. J. Bifurcation \& Chaos 15(3): 763$791,2005$.

[17] Lars Elden, Linde Wittmeyer-Koch, Hans Bruun Nielsen, 'Introduction To Numerical Computation', Studentlitteratur AB, Lund, 2004. 